Original Article

\title{
Establishment and characterization of a novel anorectal melanoma cell line derived from primary human rectal tumor
}

Running title: A novel anorectal cancer cell line

Seiichi Shinji ${ }^{1}$, Yuuki Shichi ${ }^{2}$, Takeshi Yamada $^{1}$, Goro Takahashi ${ }^{1}$, Ryo Ohta ${ }^{1}$, Hiromichi Sonoda $^{1}$, Akihisa Matsuda ${ }^{1}$, Kazuhide Yonaga $^{1}$, Takuma Iwai ${ }^{1}$, Kohki Takeda ${ }^{1}$, Koji Ueda $^{1}$, Sho Kuriyama ${ }^{1}$, Toshimitsu Miyasaka ${ }^{1}$, Yoshibumi Ueda ${ }^{3}$, Norihiko Sasaki ${ }^{4}$, Kimimasa Takahashi ${ }^{5}$, Ryuji Ohashi ${ }^{6}$, Toshiyuki Ishiwata ${ }^{2}$, Tomio Arai $^{7}$, Hiroshi Yoshida $^{1}$

${ }^{1}$ Department of Gastrointestinal and Hepato-Biliary-Pancreatic Surgery, Nippon Medical

School, 1-1-5 Sendagi, Bunkyo-ku, Tokyo 113-8603, Japan 
${ }^{2}$ Division of Aging and Carcinogenesis, Research Team for Geriatric Pathology, Tokyo Metropolitan Institute of Gerontology, 35-2 Sakae-cho, Itabashi-ku, Tokyo 173-0015, Japan

${ }^{3}$ Department of Chemistry, School of Science, The University of Tokyo, Tokyo 1130033, Japan.

${ }^{4}$ Research Team for Geriatric Medicine (Vascular Medicine), Tokyo Metropolitan Institute of Gerontology, 35-2 Sakae-cho, Itabashi-ku, Tokyo 173-0015, Japan

5 Department of Veterinary Pathology, School of Veterinary Medicine, Nippon Veterinary and Life Science University, Tokyo, 180-8602 Japan

${ }^{6}$ Integrated Diagnostic Pathology, Nippon Medical School, 1-1-5 Sendagi, Bunkyo-ku, Tokyo 113-8602, Japan.

${ }^{7}$ Department of Pathology, Tokyo Metropolitan Geriatric Hospital and Institute of Gerontology, 35-2 Sakae-cho, Itabashi-ku, Tokyo 173-0015, Japan 


\section{Corresponding Author:}

Dr. Seiichi Shinji

Department of Gastrointestinal and Hepato-Biliary-Pancreatic Surgery

Nippon Medical School, 1-1-5 Sendagi, Bunkyo-ku, Tokyo 113-8603, Japan

Phone: +81-3-3822-2131 (ext. 6752)

Fax: +81-3-5685-0989

E-mail: $\underline{\text { s-shinji@@nms.ac.jp }}$ 


\section{Abstract}

Background: Anorectal melanoma is a rare disease with poor prognosis and nonspecific symptoms, leading to difficulties in preoperative diagnosis. Here, we describe the establishment of MELS, a novel anorectal melanoma cell line derived from tumor resection of the rectum in a 40-year-old Japanese man.

Methods: Histological, electron microscopic, and immunohistochemical features of S100, HMB-45, Melan-A, and NSE positivity in the patient's tumor were confirmed as typical of surgically resected anorectal melanoma.

Results: MELS cells are round or oval cells with sharp thorn-like protrusions on some or all of the cell membrane. They form irregular attached colonies with numerous floating cells in two-dimensional culture. Transmission electron microscopy revealed that some MELS cells possess cytoplasmic melanosomes. Immunocytochemically, MELS cells showed the same staining patterns as surgical tissues. MELS cells had lower growth rates than Caco-2 (a colon adenocarcinoma cell line) and A375 (a cutaneous melanoma cell line) cells. Oxaliplatin and irinotecan were more effective in MELS cells than in Caco-2 and A375 cells. 
Conclusions: Prior to our study, no reports had provided detailed clinical information on anorectal melanoma cell lines. Thus, MELS cells should improve current knowledge on the biological behaviors of anorectal melanoma, while providing a novel platform for examining the effects of anorectal therapies.

Keywords; anorectal melanoma, novel cell line, colorectal cancer, electron microscopy, HMB-45 


\section{Introduction}

Melanoma can arise at any site where melanocytes exist, although the most common site of melanoma is the cutaneous, followed by the ocular and mucous membrane site $^{1}$. Of the mucosal melanomas, anorectal melanoma ( $<25 \%$ prevalence) is the second most common ${ }^{1}$, though it accounts for less than $1 \%$ of all melanomas and approximately $0.5-4 \%$ of rectal and anal tumors ${ }^{2-4}$. However, incidence of this cancer is increasing in certain regions, such as the United States ${ }^{5}$. Five year survival rate of anorectal melanoma has a 5-year survival rate of only $10-20 \%{ }^{6,7}$, very low compared with the $80 \%$ mean survival rate of cutaneous melanomas ${ }^{1}$.

Currently, anorectal melanomas have no specific pathologic staging system ${ }^{8}$. A recent large retrospective study on this cancer therefore used the simplified three-tiered system for head and neck melanomas, which categorizes disease into clinically localized (Stage I), regional lymph node involvement (Stage II), and distant metastasis (Stage III) ${ }^{9}$. Researchers found that these stages correlated with outcome; the 5-year survival rates of anorectal melanoma at stages I, II, and III were $26 \%, 9.8 \%$, and $0 \%$, respectively ${ }^{10}$. 
Diagnosis of anorectal melanoma is difficult because up to $80 \%$ of lesions lack obvious pigmentation, and up to $20 \%$ are histologically amelanotic; moreover, symptoms are usually nonspecific ${ }^{11-13}$. Owing to this difficulty, extremely few anorectal melanoma cell lines have been reported. We are aware of SK-MEL-246 (Memorial Sloan Kettering Cancer Center) from a metastatic rectal tumor site in a patient with melanoma, but clinical information, including sex and age, is lacking.

In this study, we established a novel human anorectal melanoma cell line (MELS)

derived from a 40-year-old Japanese man. In addition, we examined the immunohistochemical and electron microscopic features of this cell line, along with how it is affected by colorectal cancer drugs. To the best of our knowledge, this report is the first cell line established from primary rectal-derived melanoma cells.

\section{Materials and Methods}

\section{Original tumor processing}

The origin of MELS was a tumor resected from the rectum of a 40-year-old Japanese man, referred to Nippon Medical School Chiba Hokusoh Hospital in 2005 with 
anal pain and bleeding. Digital examination revealed a hard mass, and a barium enema test demonstrated a filling defect in the rectum. Colonoscopy identified an ulcerated tumor, and biopsy of the lesion showed the proliferation of epithelioid cells with pleomorphic features. Abdominoperineal resection was performed to reveal a brown to black tumor $(45 \times 50 \mathrm{~mm})$ with ulceration at the top. Tumor tissues exhibited vascular and lymph vascular invasion, as well as regional lymph node metastases. Immunohistochemical staining and transmission-electron-microscopic analysis of the resected tumor revealed characteristic features of melanoma. The patient was diagnosed with stage III anorectal melanoma with distant metastasis to the liver ${ }^{2}$. Detailed clinical information was previously reported ${ }^{14}$.

With the written informed consent of the patient and his family, a portion of the tumor was processed for primary culturing to establish a rectal melanoma cell line. This study was conducted in accordance with the Declaration of Helsinki, 2013. The Ethics Committee and Institutional Review Board at the Nippon Medical School approved all procedures (registration no. 26-03, 2014).

\section{Primary in vitro culture}


After a rinse using sterile phosphate-buffered saline (PBS) supplemented with benzylpenicillin potassium $(100 \mathrm{U} / \mathrm{mL})$ and kanamycin sulfate, the tumor sample was cut into $1 \mathrm{~mm}$ fragments. Fragments were suspended in $20 \%$ fetal bovine serum (FBS; Nichirei Bioscience Inc., Tokyo, Japan) and seeded into $60 \mathrm{~mm}$ primary tissue culture dishes that were incubated in a humidified incubator at $37^{\circ} \mathrm{C}$ in $5 \% \mathrm{CO}_{2}$ for $4 \mathrm{~h}$. Dishes were coated with 3-4 mL of Roswell Park Memorial Institute (RPMI)-1640 medium (Thermo Fisher Scientific, Waltham, MA, USA) containing 15\% FBS and antibiotics. This medium was replaced every $3 \mathrm{~d}$. When fibroblast cell growth was observed, an essentially pure tumor cell population was extracted via differential trypsinization, followed by five to six passages. The new cell line was cultured for more than 60 passages in RPMI-1640 medium containing 10\% FBS.

\section{Immunohistochemical and immunocytochemical analyses}

Paraffin-embedded tissue sections $(3.5 \mu \mathrm{m})$ were immunostained using Histofine Simple Stain Max PO (R) or (M) kits (Nichirei Bioscience Inc., Tokyo, Japan). Sections were incubated with $0.3 \%$ hydrogen peroxide in methanol for $30 \mathrm{~min}$, to block endogenous peroxidase activity. Slides were then incubated for $20 \mathrm{~h}$ at $4^{\circ} \mathrm{C}$ in $1: 1000$ 
rabbit anti-S-100 (Z0311; DAKO), 1:200 mouse anti-Pmel17/GP100 (HMB-45, M0634;

DAKO), 1:200 mouse anti-Melan A (M7196; DAKO), 1:1000 mouse anti-NSE (M0873;

DAKO), 1:150 mouse anti-cytokeratin (M0821; DAKO), or 1:2000 anti-chromogranin A (A0430; DAKO) antibodies. Bound antibodies were detected using Simple Stain Max PO (R) or (M) with diaminobenzidine tetrahydrochloride (DAB) as the substrate and counterstained with Mayer's hematoxylin for visualization. Negative controls were not stained with primary antibodies.

For immunocytochemical analysis, MELS cells were fixed using 4\% paraformaldehyde/PBS for $10 \mathrm{~min}$ and treated with $0.2 \%$ TritonX-100/PBS for $5 \mathrm{~min}$.

Cells were incubated with primary and secondary antibodies, then stained as described for the tissue sections.

\section{Human colon and melanoma cell lines}

Caco-2, a conventional human colon cancer cell line, was obtained from Riken BRC Cell Bank (Ibaraki, Japan), and cultured in 10\% FBS-supplemented RPMI-1640 media at $37^{\circ} \mathrm{C}$ in $5 \% \mathrm{CO}_{2}$. Human melanoma cell line $\mathrm{A} 375$ was obtained from American 
Type Culture Collection (ATCC) and cultured under the same conditions in 10\% FBSsupplemented DMEM.

Scanning electron microscopy (SEM)

MELS were fixed overnight with $2.5 \%$ glutaraldehyde in $0.1 \mathrm{M}$ phosphate buffer $(\mathrm{pH} 7.4)$ at $4^{\circ} \mathrm{C}$. Glutaraldehyde was then removed, and cells were washed with PBS. Cells were post-fixed with osmium tetroxide for $30 \mathrm{~min}$ to prevent sphere samples from collapsing during sample preparation. After complete dehydration via a graded ethanol series, spheres were suspended in $100 \%$ ethanol, air-dried, and covered with a platinum layer using an MSP-1S sputter coater (Shinku Device, Ibaraki, Japan). Cells were examined and photographed using a Phenom Pro desktop scanning electron microscope with reflective or secondary electrons (Thermo Fisher Scientific).

\section{Transmission electron microscopy (TEM)}

Rectal tumor tissues and MELS cells were fixed with 2.5\% glutaraldehyde in 0.1 $\mathrm{M}$ phosphate buffer ( $\mathrm{pH} 7.4$ ), then post-fixed for $1 \mathrm{~h}$ with $2 \% \mathrm{OsO}_{4}$ dissolved in distilled water. Samples were dehydrated using an ethanol gradient, embedded in Epon, and sliced into ultrathin sections using an ultramicrotome. Sections were then stained with uranyl 
acetate and lead citrate for examination under a transmission electron microscope $(\mathrm{H}-$ 7500; Hitachi High-Technologies, Tokyo, Japan).

\section{Anti-drug resistance assays}

Cells $\left(3.0 \times 10^{3}\right.$ per well) were plated in 96-well culture dishes containing growth medium. Each anti-cancer drug was administered at the indicated concentrations after 1 d. Four days later, cell growth rates were measured with ATP assays, performed using CellTiter-Glo ${ }^{\circledR} 2.0$ (Promega, Madison, USA). Cell viability was calculated as percentage of luminescence in drug-treated cells versus in untreated control cells.

\section{Statistical analyses}

Data were analyzed in Easy R (EZR; Saitama Medical Center, Jichi Medical University, Saitama Japan), a GUI for R (The R Foundation for Statistical Computing, Vienna, Austria) that adds functions frequently used in biostatistics. Continuous variables were analyzed using the student's t-test. Significance was set at $\mathrm{P}<0.05$.

\section{Results}


Tumor cells in the rectum formed a solid structure (Fig. 1A). Cells possessed atypical nuclei and numerous atypical mitoses (Fig. 1B, arrows); most exhibited epithelioid features and partial-spindle appearance. Tissue sections had less than $10 \%$ of tumor cells, which contained small dark brown pigment localized to the cytoplasm (Fig. 1C). These were revealed to be Stage IV melanosomes under TEM (Fig. 1D, arrows).

Immunohistochemical analysis revealed that tumor cells were positive for S-100, HMB-45, Melan-A, and NSE, but negative for cytokeratin and chromogranin A (Fig. 2). Cytokeratin was localized in normal epithelial cells, while chromogranin A was localized in neuroendocrine cells of normal ducts. Based on these morphological characteristics, the tumor was diagnosed as a melanoma in the rectum.

\section{Establishment of MELS}

MELS was weakly attached to culture plates and formed irregularly shaped colonies (Fig. 3A), with sharp thorns at the periphery (Fig. 3B, arrows). Morphology was variable, including long protrusions (Fig. 3C, arrows) and a semi-circular shape with short protrusions on the cell margin (Fig. 3D, arrows). Observations under SEM showed that colonies comprised round to oval-shaped MELS cells (Fig. 4A, B), with peripheral cells 
possessing protrusions of various lengths (Fig. 4C, D). Furthermore, TEM analysis showed that MELS cells had numerous short-to-long protrusions (Fig. 5A), and stage I and II premelanosomes were present in their cytoplasm, (Fig. 5B white and black arrows respectively). Immunocytochemical analysis indicated that MELS cells cultured in chamber slides were positive for S-100, HMB-45, Melan-A, and NSE, but negative for cytokeratin and chromogranin A (Fig. 6). Thus, established MELS cells retained properties of the original rectal melanoma tissues.

\section{Viability of MELS cells after the addition of anti-cancer drugs}

The ATP assays for comparing growth across MELS, Caco-2 (human colon adenocarcinoma line), and A375 (human cutaneous melanoma line) cells showed that growth rates were markedly lower in MELS cells than in Caco-2 and A375 cells (Fig. 7A). Next, we used viability assays to compare the effects of common anti-colorectal cancer drugs (oxaliplatin, fluorouracil [5-FU], and irinotecan) on the three cells types. After adding $10 \mu \mathrm{M}$ oxaliplatin, MELS cells had lower viability than in the other two cells. Increasing oxaliplatin concentration to $100 \mu \mathrm{M}$ resulted in lower viability for MELS and A375 cells compared with Caco-2 cells (Fig. 7B). Treatment with $100 \mu \mathrm{M}$ 5-FU also 
caused lower viability in MELS and A375 cells than in Caco-2 cells (Fig. 7B). Finally, under both 10 and $100 \mu \mathrm{M}$ irinotecan treatment, MELS and A375 cells exhibited lower viability than Caco-2 cells (Fig. 7B).

\section{Discussion}

In this study, we performed preoperative diagnosis of anorectal melanoma using colorectal biopsy, among the most accurate ways to diagnosis this cancer ${ }^{15}$. We determined that the observed tumor was a poorly differentiated adenocarcinoma with solid proliferation. Additionally, we did not observe melanin in the biopsy sections, nor did we perform immunohistochemical and TEM analyses. We chose to prepare for establishing a cultured cell line before confirming our melanoma diagnosis.

The surgically resected rectal tissues showed typical immunohistochemical staining patterns for anorectal melanoma. In the established MELS cells, we observed Stage I and II premelanosomes using TEM (classifications are according to morphology and melanin synthesis ${ }^{16}$ ). Anorectal melanoma has fewer melanin-positive cases than cutaneous melanoma; accordingly, melanin was found in less than $10 \%$ of tumor cells in our sample. 
A previous report using SEM showed that cutaneous melanoma cells possess numerous extensions on their surface, including microblebs, microvilli, and some lamella ${ }^{17}$. Several cutaneous melanoma protrusions were extremely long, slender, and twisted between neighboring cells. We observed that MELS cells form aggregated colonies, with cells exhibiting a variety of morphologies, such as semi-circular protrusions and very long, rod-like cytoplasm. In general, they were pleomorphic cell types with short to long protrusions on their surfaces. Corroborating our findings, previously published SEM data indicated that melanoma cell surfaces had numerous extensions, some with differentiations that suggested special function, although smoothsurfaced cells were also present ${ }^{17}$.

MELS cells were positive for melanoma-specific antigens, specifically anti-S-100, HMB-45, and Melan-A (also known as MART-1), while being negative for epithelial and neuroendocrine markers. Anti-S-100 protein is the most common immunohistochemical stain used for diagnosing anorectal melanoma, being highly sensitive to melanocytic differentiation $^{18}$. HMB-45 and Melan-A are melanocyte-specific markers used for the diagnosis of melanoma ${ }^{19}$. In particular, the latter two antibodies are present in most 
anorectal melanoma cases $\left(94 \%\right.$ and $93 \%$, respectively) ${ }^{18}$. MART-1/Melan-A is reportedly required for the maturation of Pme17 and HMB45 reacts with the matured Pmel17 $7^{20}$. The double positivity of MART-1/Melan-A and HMB-45 in MELS cells is reasonable.

Anorectal melanoma is characterized by aggressive behavior, poor outcome due to late diagnosis, and fast tumor growth in the rich vascular and lymphatic supply of the anorectal mucosa ${ }^{21}$. In this study, we found that MELS cells had a far lower proliferation rate than conventional colorectal cancer and cutaneous melanoma.

For patients with anorectal melanoma, treatments include surgery, radiotherapy, chemo-immunotherapy, and targeted therapy ${ }^{22}$. It is known that JAK mutation has been a major mechanism against anti-PD-L1 antibody in metastatic melanoma of the cutaneous 23. Management strategies for anorectal melanoma have been hampered by a lack of randomized trials. Tumors tend to be considerably resistant to radiotherapy and show a poor response to chemotherapy. Oxaliplatin and irinotecan are usually used for colorectal cancer treatments, but melanomas derived from colorectum are treated in the same manner as cutaneous melanomas. We evaluate the effectiveness of therapeutic drugs for 
colorectal cancer in MELS cells and found that these anti-colorectal cancer drugs were effective for MELS. Further studies are needed to clarify the underlying mechanisms and the effectiveness of these drugs in vivo.

To the best of our knowledge, SK-MEL-246 is the only other cell line that has been derived from a human rectal melanoma cell line ${ }^{24}$. However, clinical details of this cell line, including donor gender and age, are unknown. Moreover, the cell line was established from a metastatic rectal tumor site in a patient with melanoma. As far as we are aware, no reports are available concerning the immunohistochemical, electron microscopic, and genetic characteristics of SK-MEL-246 cells. Our study thus fills an important gap in anorectal melanoma research.

In conclusion, we established MELS, a new anorectal melanoma cell line derived from a 40-year-old Japanese man. This cell line expresses typical melanoma-specific antigens and possesses melanosomes in the cytoplasm. Further studies of MELS, including identification of genetic mutations, should help us better characterize anorectal melanomas and contribute to the development of anticancer drugs, immune therapy, and radiotherapy for treating this cancer. 


\section{Acknowledgments}

The authors thank Mr. Fumio Hasegawa, Ms. Yasuko Hasegawa (Tokyo Metropolitan Institute of Gerontology), Ms. Kiyoko Kawahara (Nippon Medical School), Mr. Tatsuo Oguro (Nippon Medical School Chiba Hokusoh Hospital), and Miss Junko Mieda (Nippon Medical School Chiba Hokusoh Hospital) for their excellent technical assistance, and the late Dr. Yoshiharu Ohaki (Nippon Medical School Chiba Hokusoh Hospital) for his invaluable suggestions. This study was supported in part by JSPS KAKENHI (19K11759 and 19K09207) through a Grant-in-Aid for Scientific Research (C) awarded to T. Ishiwata and N. Sasaki.

\section{Disclosure Statement}

The authors have no conflicts to declare. 


\section{References}

1. Chang AE, Karnell LH, Menck HR. The National Cancer Data Base report on cutaneous and noncutaneous melanoma: a summary of 84,836 cases from the past decade. The American College of Surgeons Commission on Cancer and the American Cancer Society. Cancer. 1998 Oct 15;83(8):1664-78.

2. Singer M, Mutch MG. Anal melanoma. Clin Colon Rectal Surg. 2006 May;19(2):78-87.

3. van Schaik PM, Ernst MF, Meijer HA, Bosscha K. Melanoma of the rectum: a rare entity. World J Gastroenterol. 2008 Mar 14;14(10):1633-5.

4. Row D, Weiser MR. Anorectal melanoma. Clin Colon Rectal Surg. 2009 May;22(2):120-6.

5. Chen H, Cai Y, Liu Y, et al. Incidence, Surgical Treatment, and Prognosis of Anorectal Melanoma From 1973 to 2011: A Population-Based SEER Analysis. Medicine (Baltimore). 2016 Feb;95(7):e2770. 
6. Longo WE, Vernava AM, 3rd, Wade TP, Coplin MA, Virgo KS, Johnson FE.

Rare anal canal cancers in the U.S. veteran: patterns of disease and results of treatment. Am Surg. 1995 Jun;61(6):495-500.

7. Hillenbrand A, Barth TF, Henne-Bruns D, Formentini A. Anorectal amelanotic melanoma. Colorectal Dis. 2008 Jul;10(6):612-5.

8. Chae WY, Lee JL, Cho DH, Yu CS, Roh J, Kim JC. Preliminary Suggestion about Staging of Anorectal Malignant Melanoma May Be Used to Predict Prognosis. Cancer Res Treat. 2016 Jan;48(1):240-9.

9. Ballantyne AJ. Malignant melanoma of the skin of the head and neck. An analysis of 405 cases. Am J Surg. 1970 Oct;120(4):425-31.

10. Iddings DM, Fleisig AJ, Chen SL, Faries MB, Morton DL. Practice patterns and outcomes for anorectal melanoma in the USA, reviewing three decades of treatment: is more extensive surgical resection beneficial in all patients? Ann Surg Oncol. 2010 Jan;17(1):40-4. 
11. Slingluff CL, Jr., Vollmer RT, Seigler HF. Anorectal melanoma: clinical characteristics and results of surgical management in twenty-four patients. Surgery. 1990 Jan;107(1):1-9.

12. Morson BC, Volkstadt H. Malignant melanoma of the anal canal. J Clin Pathol. 1963 Mar;16(2):126-32.

13. Carvajal RD, Spencer SA, Lydiatt W. Mucosal melanoma: a clinically and biologically unique disease entity. J Natl Compr Canc Netw. 2012 Mar;10(3):345-56.

14. Seya T, Tanaka N, Shinji S, et al. Case of rectal malignant melanoma showing immunohistochemical variability in a tumor. J Nippon Med Sch. 2007 Oct; $74(5): 377-81$.

15. Wang S, Sun S, Liu X, et al. Endoscopic diagnosis of primary anorectal melanoma. Oncotarget. 2017 Jul 25;8(30):50133-40.

16. Raposo G, Tenza D, Murphy DM, Berson JF, Marks MS. Distinct protein sorting and localization to premelanosomes, melanosomes, and lysosomes in pigmented melanocytic cells. J Cell Biol. 2001 Feb 19;152(4):809-24. 
17. Drzewiecki KT. The surface morphology of the melanoma cell. A scanning electron microscope study on the primary cutaneous melanoma. Scand J Plast Reconstr Surg. 1977;11(1):9-16.

18. Chute DJ, Cousar JB, Mills SE. Anorectal malignant melanoma: morphologic and immunohistochemical features. Am J Clin Pathol. 2006 Jul;126(1):93-100.

19. Atak I. Anorectal Malignant Melanoma: Retrospective Analysis of Six Patients and Review of the Literature. Prague Med Rep. 2018;119(2-3):97106.

20. Hoashi T, Muller J, Vieira WD, et al. The repeat domain of the melanosomal matrix protein PMEL17/GP100 is required for the formation of organellar fibers. J Biol Chem. 2006 Jul 28;281(30):21198-208.

21. Meguerditchian AN, Meterissian SH, Dunn KB. Anorectal melanoma: diagnosis and treatment. Dis Colon Rectum. 2011 May;54(5):638-44.

22. Malaguarnera G, Madeddu R, Catania VE, et al. Anorectal mucosal melanoma. Oncotarget. 2018 Feb 2;9(9):8785-800. 
23. Asano T. Drug Resistance in Cancer Therapy and the Role of Epigenetics. J Nippon Med Sch. 2020 Dec 14;87(5):244-51.

24. Fogh J, Fogh JM, Orfeo T. One hundred and twenty-seven cultured human tumor cell lines producing tumors in nude mice. J Natl Cancer Inst. 1977 Jul;59(1):221-6. 


\section{Figure legends}

Figure 1. Histological and transmission electron microscopic (TEM) features of surgically resected rectal tumor tissues

Hematoxylin and eosin staining showing that melanoma cells formed solid tumors with atypical mitoses (arrows in B) and brown melanin pigment in their cytoplasm (A-C). TEM revealed Stage IV melanosomes in the cytoplasm (D, arrows). Original magnification: A $\times 40 ; \mathrm{B}, \mathrm{C} \times 600$. D: Scale bar $=1 \mu \mathrm{m}$

Figure 2. Immunohistochemical analysis of surgically resected rectal tumor tissues Rectal tumor cells were positive for S-100, HMB-45, Melan-A, and NSE, but negative for cytokeratin and chromogranin A. Original magnification: $\times 200$

\section{Figure 3. Phase-contrast images of MELS cells}

MELS cells were cultured in two-dimensional conditions. Cells formed irregular colonies with sharp protrusions (arrows in A and B). Morphology was varied, with long protrusions or a semi-circular appearance (arrows in C and D). A: Scale bar $=200 \mu \mathrm{m}$, $\mathrm{B}-\mathrm{D}=50 \mu \mathrm{m}$

Figure 4. Scanning electron microscopic (SEM) analysis of MELS cells 
MELS cells formed grape-like colonies with short to long protrusions at the periphery (A-D).

Scale bar: $A=100 \mu \mathrm{m}, \mathrm{B}-\mathrm{D}=10 \mu \mathrm{m}$. A, B: reflective electrons, $10 \mathrm{kV}$; C, D: secondary electrons, $5 \mathrm{kV}$.

Figure 5. TEM analysis of MELS cells

MELS cells had short to long protrusions (A) and premelanosomes (B, Stage I: white arrows, Stage II: black arrows) in the cytoplasm. M: mitochondria, Scale bar: $\mathrm{A}=2 \mu \mathrm{m}$, $\mathrm{B}=500 \mathrm{~nm}$

\section{Figure 6. Immunocytochemical analysis of MELS cells}

MELS cells were positive for S-100, HMB-45, Melan-A, and NSE, but negative for cytokeratin and chromogranin A. Original magnification: $\times 600$

Figure 7. Anti-cancer drug resistance assay in MELS, Caco-2, and A375 cells

ATP assays compared growth across MELS, Caco-2 (human colon adenocarcinoma cell line), and A375 cells (human cutaneous melanoma cell line) (A). Dose response (10 or $100 \mu \mathrm{M})$ of MELS, Caco-2, and A375 cells to oxaliplatin, 5-FU, and irinotecan was determined using ATP assays (B). ${ }^{*} p<0.05, * * p<0.01$. 
Fig. 1

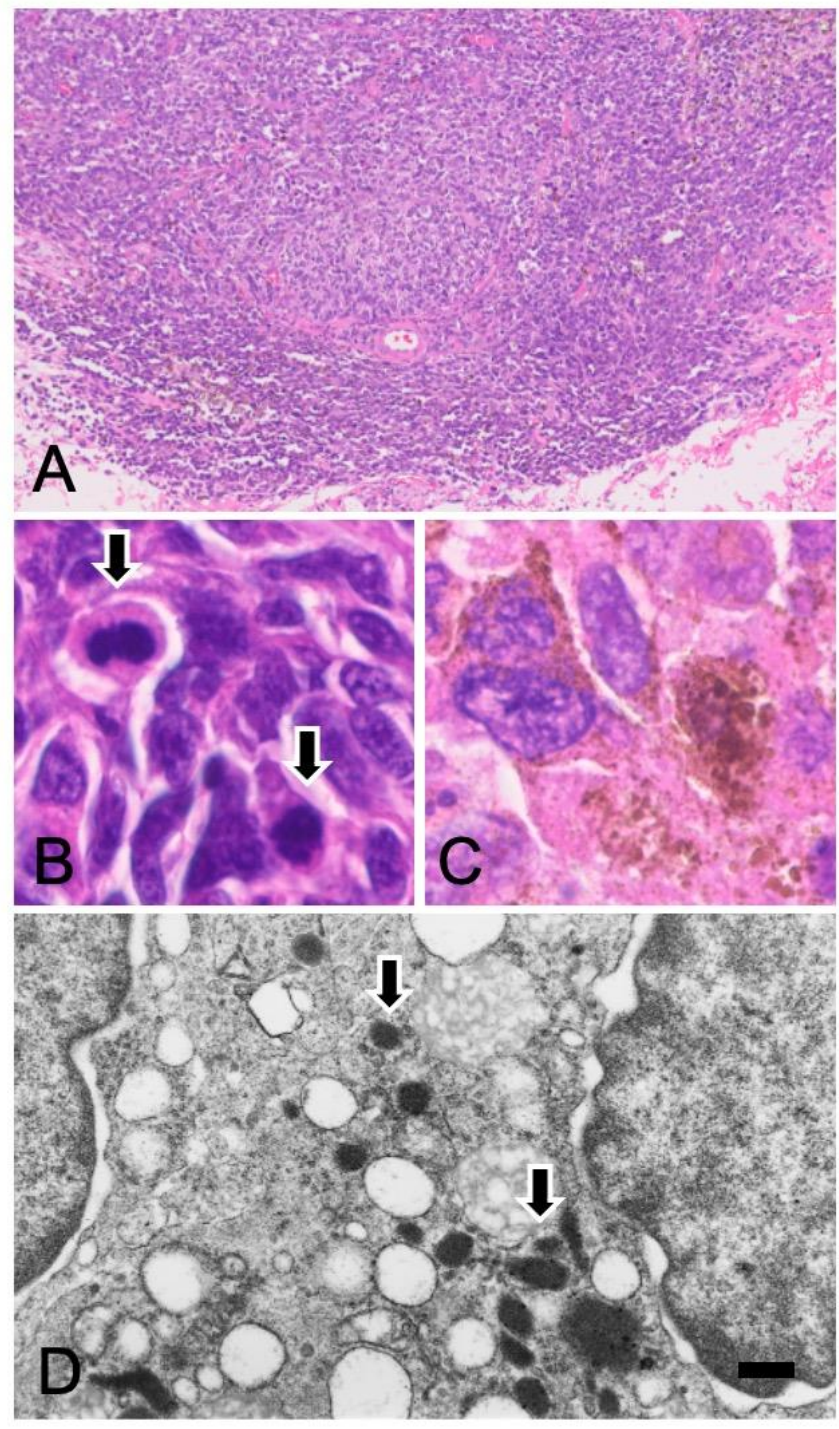


Fig. 2

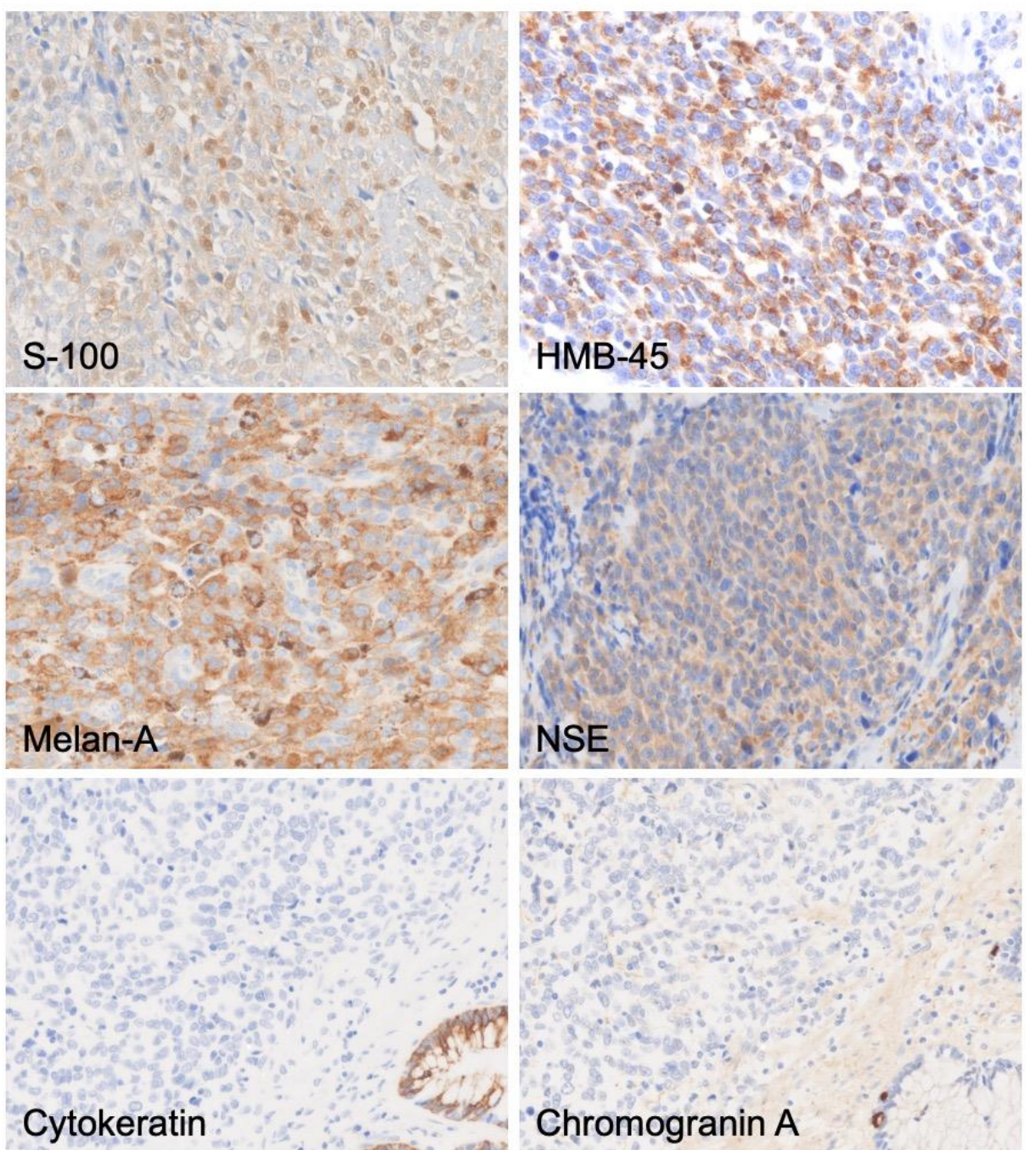


Fig. 3

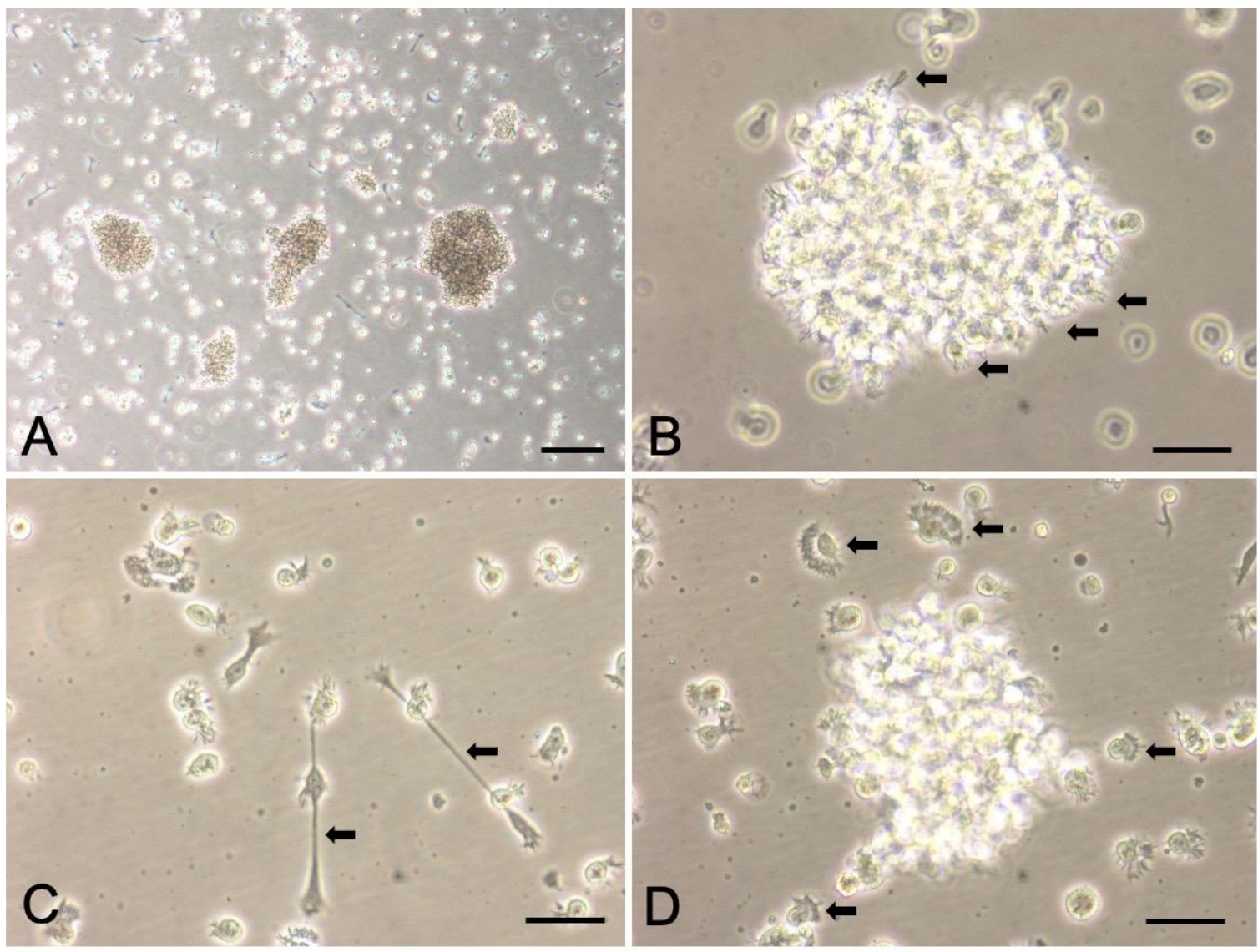


Fig. 4

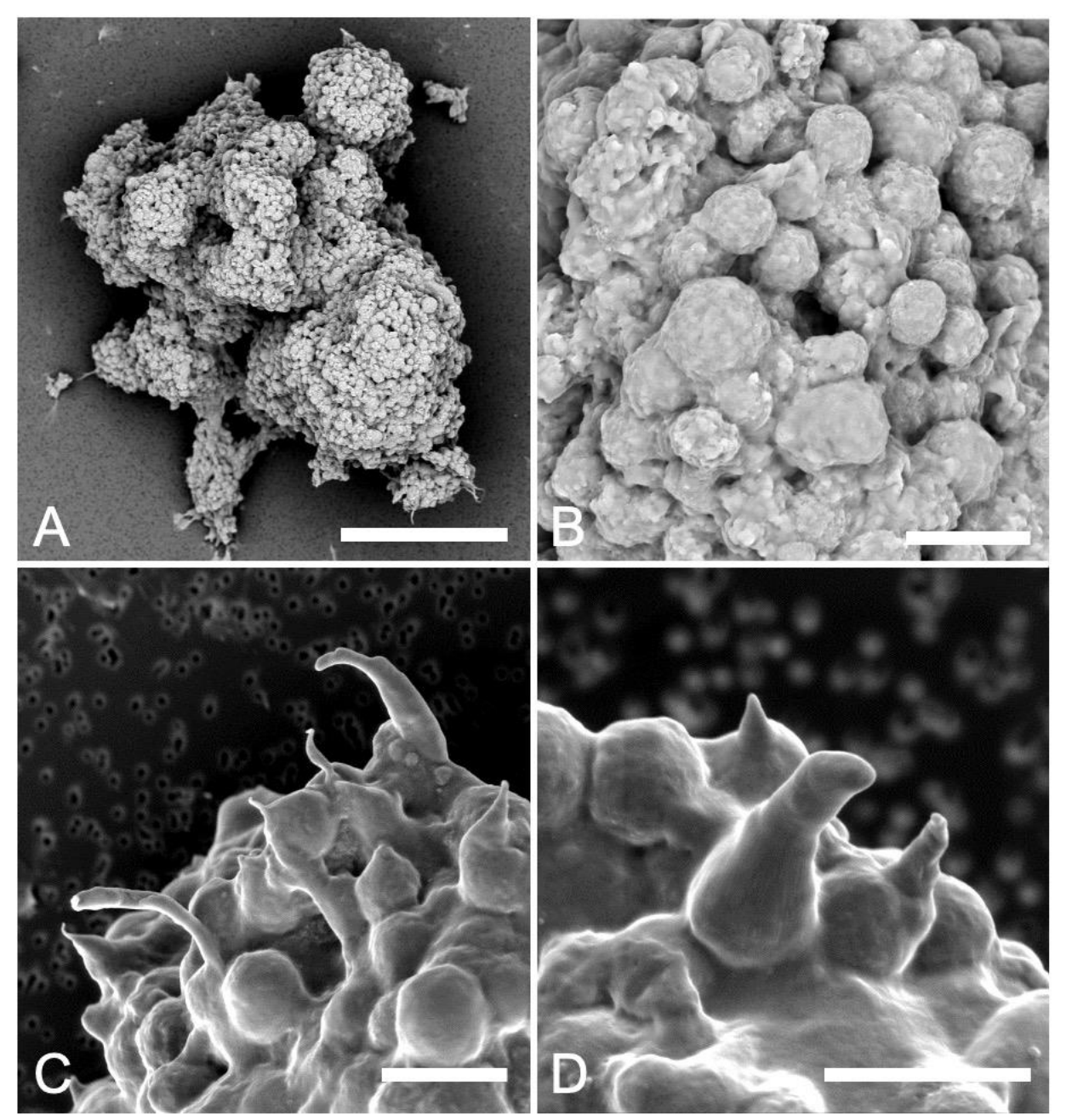


Fig. 5

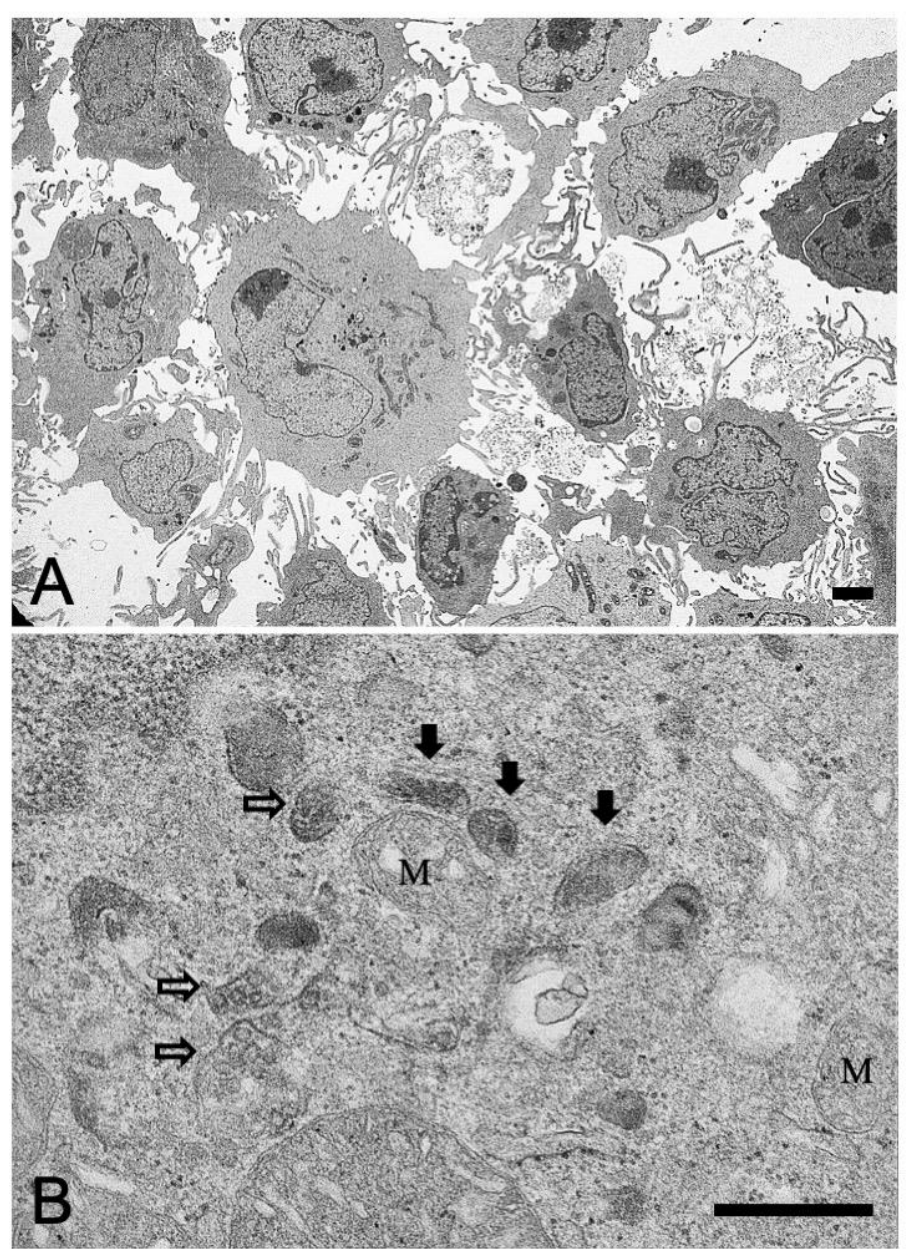




\section{Fig. 6}

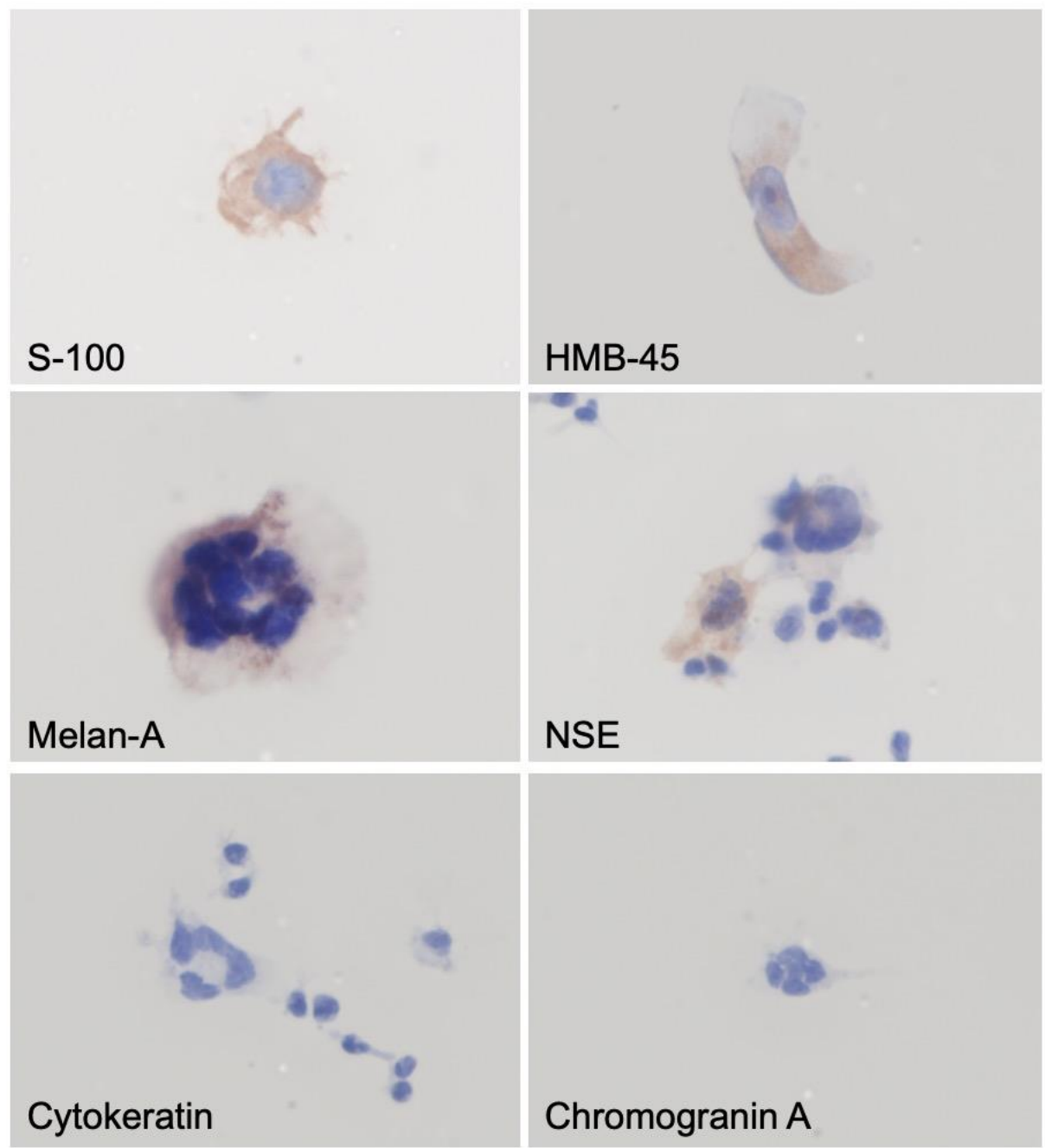


Fig. 7

A

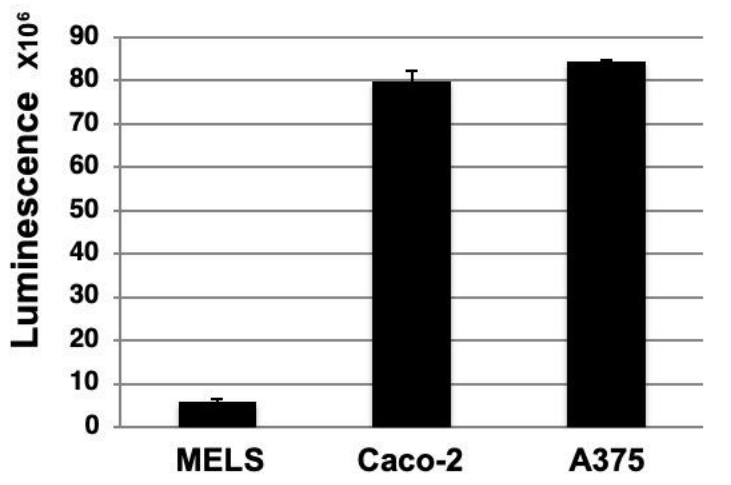

B
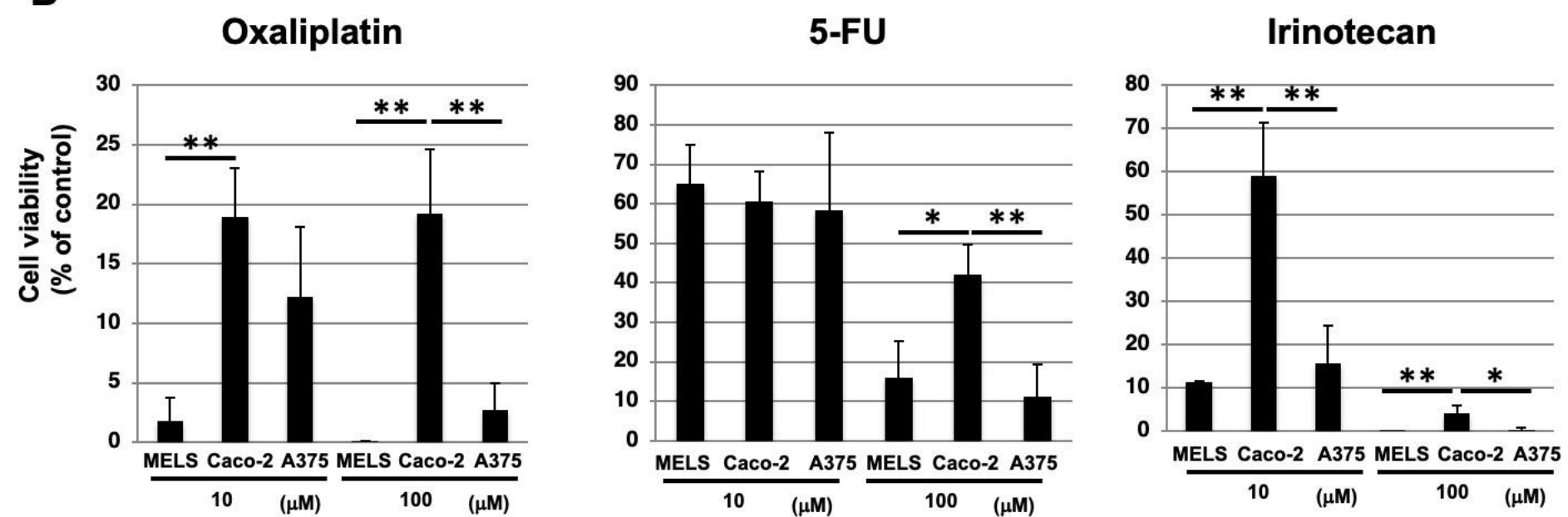